\title{
Chronic Patients' Activation and Its Association with Stress, Anxiety, Depression, and Quality of Life: A Survey in Southeast Iran
}

\author{
Mohammad Ali Zakeri $\mathbb{D}^{1,2}$ Mahlagha Dehghan $\mathbb{D}^{1},{ }^{3}$ Fatemeh Ghaedi-Heidari $\left(\mathbb{D},{ }^{4}\right.$ \\ Maryam Zakeri $(1)^{5}$ and Gholamreza Bazmandegan $\mathbb{1}^{6,7}$ \\ ${ }^{1}$ Non-Communicable Diseases Research Center, Rafsanjan University of Medical Sciences, Rafsanjan, Iran \\ ${ }^{2}$ Social Determinants of Health Research Center, Rafsanjan University of Medical Sciences, Rafsanjan, Iran \\ ${ }^{3}$ Nursing Research Center, Kerman University of Medical Sciences, Kerman, Iran \\ ${ }^{4}$ Faculty of Nursing and Midwifery, Isfahan University of Medical Sciences, Isfahan, Iran \\ ${ }^{5}$ Physiology-Pharmacology Research Center, Research Institute of Basic Medical Sciences, Rafsanjan University of Medical Sciences, \\ Rafsanjan, Iran \\ ${ }^{6}$ Clinical Research Development Unit, Ali-Ibn Abi-Talib Hospital, Rafsanjan University of Medical Sciences, Rafsanjan, Iran \\ ${ }^{7}$ Department of Family Medicine, Ali-Ibn Abi-Talib Hospital, School of Medicine, Rafsanjan University of Medical Sciences, \\ Rafsanjan, Iran
}

Correspondence should be addressed to Gholamreza Bazmandegan; bkhrbster@gmail.com

Received 6 November 2020; Revised 6 March 2021; Accepted 18 March 2021; Published 26 March 2021

Academic Editor: Yeong Shiong Chiew

Copyright ( 2021 Mohammad Ali Zakeri et al. This is an open access article distributed under the Creative Commons Attribution License, which permits unrestricted use, distribution, and reproduction in any medium, provided the original work is properly cited.

\begin{abstract}
A better perception of the factors associated with patient activation, as a way to improve self-management, is the most important step in planning patient-centered education for chronic disease management. Therefore, the present study is aimed at investigating the relationship between activation, stress, anxiety, depression, and quality of life (QOL) in patients with chronic diseases. This correlational study was performed on 293 chronic patients admitted to coronary care units (CCUs) in one of the hospitals in Rafsanjan. The Patient Activation Measure (PAM), Quality of Life-BREF (WHOQOL-BREF), and Depression, Anxiety, and Stress Scale-21 Items (DASS-21) were used to collect data. The data were then analyzed using SPSS 22. A significant positive correlation was observed between general QOL and PAM $(P<0.001)$. In addition, a significant negative correlation was found between PAM, stress $(P=0.032)$, and depression $(P=0.025)$. The results of multivariate linear regression indicated that only physical and psychological subscales of QOL significantly predicted PAM $(B=0.24 ; 95 \%$ confidence interval; $P$ value $<0.05)$. Owing to the fact that some subscales of QOL have a determinant role in the PAM of chronic patients, healthcare providers are recommended to plan and implement the necessary interventions to improve the QOL and the health outcomes of chronic patients.
\end{abstract}

\section{Introduction}

The silent pandemic of chronic diseases, one of the biggest public health challenges worldwide, is gradually spreading to all countries [1]. Chronic diseases have a high mortality rate and impose a heavy burden on healthcare systems [2]. According to the Institute of Health Metrics and Evaluation (IHME), chronic diseases accounted for $72 \%$ of the causes of death in 2016 [1]. According to the World
Health Organization (WHO), chronic diseases caused 73 percent of deaths and $60 \%$ of the global burden of disease in 2020. In addition, $79 \%$ of these deaths will occur in developing countries [3]. The most common chronic diseases, including cardiovascular disease, cancer, chronic obstructive pulmonary disease (COPD), and type 2 diabetes have common and preventable risk factors such as hypertension, weight gain, and high-risk behaviors such as poor nutrition, sedentary lifestyle, and smoking [4]. 
According to the IHME report, ischemic heart disease was the leading cause of all deaths in the world and Iran in 2017 [5].

Self-management is one way to reduce the burden of disease on both the patient and the healthcare system and to reduce mortality of patients with chronic disease, which is one of the important factors involved [2]. Patient activation is one of the methods used to improve self-management. Patient activation refers to the knowledge, skills, and confidence in controlling one's health [6], which reflects the patient's perception of his/her role in the healthcare and selfmanagement program [7]. The concept of patient activation has been demonstrated to correlate with improved clinical outcomes, increased preventative care, and decreased healthcare-related cost. This concept refers to the actions and activities performed by the patient to improve his/her illness. Patients being activated to manage their conditions had a positive effect on self-management and improved health outcomes $[8,9]$. Assessing patient activation after a lifethreatening illness provides opportunities for healthcare providers to develop care plans tailored to the patients' needs before their discharges [10].

In the last two decades, various studies have examined different populations by using the patient activation method. These studies show that patient activation is one of the effective factors in improving health-related behaviors and outcomes in patients with chronic diseases [1113]. Greene and Hibbard showed that activation of patients with chronic diseases is associated with reduced admission to the emergency department, obesity, and smoking. In addition, patient activation minimizes the chance of breast cancer diagnosis in screening tests and abnormal changes in blood tests such as cholesterol, triglyceride, and glycosylated hemoglobin $\mathrm{A}_{1} \mathrm{C}$ [11]. One study found that chronically ill patients with lower levels of activation imposed higher costs on healthcare systems [12]. Furthermore, the study performed on patients with COPD showed a significant negative correlation between patients' activation, current smoking, hospital anxiety and depression scores, and respiratory symptoms [13]. Another study on patients with multiple sclerosis showed that patient activation is positively correlated with self-efficacy and academic achievement, but it had no significant relationship with QOL [14]. One study showed that patient activation increased satisfaction with postoperative outcomes in patients with lumbar and cervical spine diseases [15].

These studies have used a variety of methodologies and have mainly focused on parameters related to specific diseases. The present study assumed that many patients suffered from more than one chronic disease, and it did not emphasize a specific chronic disease. In addition, a better perception of some important variables (such as mental status and QOL) associated with patient activation is the most important step in planning patient-centered education for chronic disease management. Therefore, the present study is aimed at investigating the relationship between patient activation, stress, anxiety, depression, and QOL in patients with chronic diseases.

\section{Methods}

2.1. Study Design and Participants. This cross-sectional correlational study was conducted on 293 chronic patients admitted to the Cardiac Care Unit (CCU) and medical wards in Ali Ibn Abitaleb Hospital of Rafsanjan. Data were collected from January to April 2019. The inclusion criteria were chronically ill patients aged more than 18 years old, without known psychological problems (depression, bipolar disorder) and acute cognitive disorders. Patients with visual and auditory processing disorders were excluded from the study.

2.2. Study Setting. Iran is a collection of people with diverse languages and cultures. In general, the growth of urbanization in Iran is increasing, and the population in all cities has not grown evenly, but mainly large cities have grown faster than small cities. Being in the vicinity of the desert, southern and southeastern cities of Iran are less developed and economically grown and have brought about more problems and challenges for patients. For this reason, the QOL of people living in southeastern cities of Iran is different from those living in other places [16].

2.3. Sample Size and Sampling. Based on studies by Green et al. [17] to determine the relationship between activation, anxiety, stress, depression, and QOL $(r=0.21)$ with $99 \%$ confidence and $90 \%$ test power, the sample size was considered 240 people according to the following formula:

$$
\begin{gathered}
\omega=\frac{1}{2} \operatorname{Ln} \frac{1+r}{1-r}, \\
n=\frac{\left(Z_{1-\alpha / 2}+Z_{1-\beta}\right)^{2}}{(\omega)^{2}}+3 .
\end{gathered}
$$

Concerning the conditions of chronic patients and the possibility of dropout, we examined 300 chronic patients according to inclusion criteria, and all of them wanted to participate in the study, so 300 questionnaires were collected. Out of these 300 questionnaires, seven questionnaires were excluded from the study due to deficiencies in completion, and finally, 293 were included in the analysis (Figure 1). The response rate was $97.66 \%$.

\subsection{Measurement}

2.4.1. Demographic Information. Demographic information of the participants included age, sex, body mass index (BMI), marital status, occupation, education level, income, number of hospital stays, presence of other diseases, drug use, and type of chronic disease.

2.4.2. Patient Activation Measure-13. The American short form of Patient Activation Measure-13 (PAM-13) was developed by Hibbard et al. to examine self-management [6]. Activation therapy assesses the patient's knowledge, skills, beliefs, and confidence in health management and healthcare. This measure consists of 13 items on the Likert scale ranging from one (strongly disagree) to four (strongly agree). Answers are calculated based on the standard metric system converted 


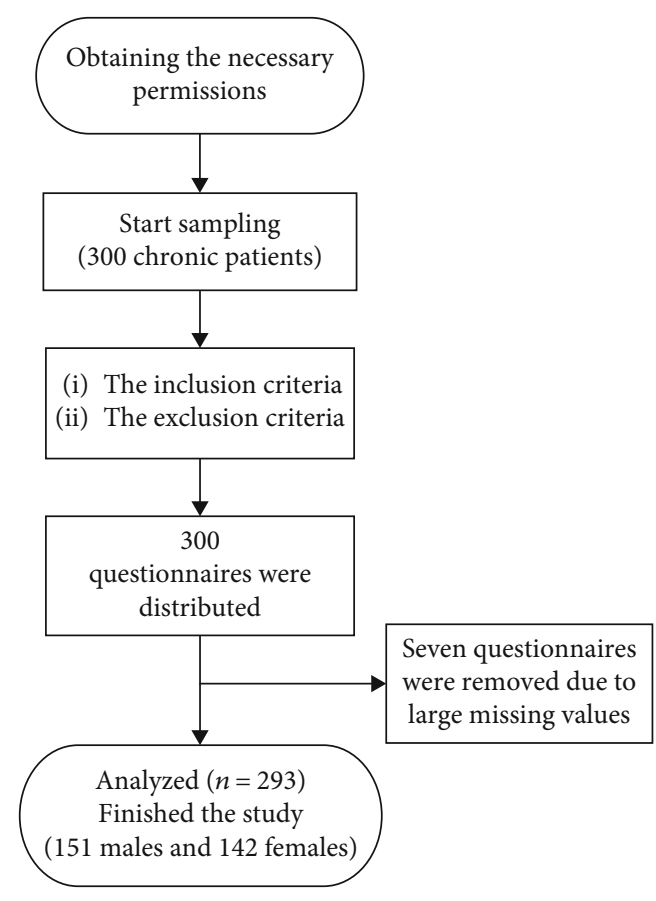

FIgURE 1: The study flowchart.

from zero to 100 (zero = the lowest activation level, $100=$ the highest level), and the score of the questionnaire varies from 0 to 100 , with higher scores reflecting higher levels of patient activation. The validity of this questionnaire was obtained by using face and content validities. We used internal consistency and test-retest for the PAM questionnaire to assess reliability. The internal consistency was good $(\alpha=0.88)$, and the intraclass correlation coefficient (ICC) was 0.96 .

2.4.3. WHOQOL-BREF. This questionnaire has been used by the WHO to measure the QOL of individuals in the last two weeks. This self-report questionnaire with 4 domains and 26 items examines the health status and QOL. The physical health domain, psychological domain, social relationship domain, and environmental domain are assessed in this questionnaire. It has also two questions for assessment of overall QOL and general health. The score of each item is on the scale value of 1 (never) to 5 (very high). Items 3, 4, and 25 are scored reversely. For this scale to be interpreted, the short version must be converted into a long version, and then, the QOL in each domain must be interpreted from zero to 100. It is noteworthy that the WHOQOL-BREF questionnaire does not allow a single QOL score. The higher scores indicate a better QOL. Nejat et al. confirmed the validity and reliability of the questionnaire in Iran in 2006. Cronbach's alpha coefficient of 0.78 was obtained for the questionnaire [18].

2.4.4. Depression, Anxiety, and Stress Scale-21 Items (DASS21). The Depression, Anxiety, and Stress Scale-21 Items (DASS-21) was developed by Lovibond and Lovibond in 1995 to assess the psychological constructs of depression, anxiety, and stress [19]. The scale consists of 21 items with three subscales of depression, anxiety, and stress (each subscale includes seven items) on a four-point Likert scale (never/low/medium/high). The lowest score is zero, and the highest score is three. The final score of each is obtained through the sum of the scores of the related items. The final score of the subscales should be doubled. Samani and Joukar examined the validity and reliability of this scale in Iran and reported the retest validity to be $0.80,0.76$, and 0.77 for depression, anxiety, and stress, respectively. Cronbach's alpha coefficient was reported to be $0.81,0.74$, and 0.78 , for depression, anxiety, and stress, respectively [20].

2.5. Data Collection and Analysis. After obtaining the necessary permissions, the researcher referred to the research settings and started sampling. Thus, the demographic information questionnaire, Patient Activation Measure-13, DASS, and WHOQOL-BREF were distributed among the eligible samples, who answered the questionnaires in the presence of the researcher. One researcher explained to the patients how to complete the questionnaires. According to the instructions for completing the questionnaire, patients were asked to complete the questions according to the last two weeks.

The data were analyzed by SPSS 22. Descriptive statistics (frequency, percentage, mean, and standard deviation) were used to describe the participants' characteristics. The Kolmogorov-Smirnov test was done to check the normal distribution of the quantitative data. Spearman and Pearson correlation coefficients were used to determine the correlation between the study quantitative variables. The independent $t$-test, Mann-Whitney $U$, analysis of variance, and Kruskal-Wallis tests were used to determine PAM-13 scores according to the qualitative variables. Multivariate linear regression with the enter method was used to identify the PAM-13 determinants. A significance level of 0.05 was considered.

2.6. Ethical Considerations. This research has a code of ethics No. IR.RUMS.REC.1397.109 from the University of Medical Sciences. Before sampling, informed written consent was taken from chronic patients, who were explained about the objectives of the study, confidentiality and anonymity of the information, and the voluntary participation in the study and voluntary withdrawal at any time. Participants with major anxiety, stress, and depression referred to a hospital psychiatrist for further evaluation. They also explained the consequences and problems of increasing anxiety, stress, and depression.

\section{Results}

The mean age of participants was $63.18 \pm 13.44$ years. The majority of the participants were male (51.5\%), married (86.0\%), and illiterate $(50.2 \%)$ and had no history of hospital stay $(37.2 \%)$ (Table 1$)$.

The mean score of PAM-13 was $56.99 \pm 15.32$, which was greater than the midpoint of the questionnaire $($ score $=50)$. The mean scores of anxiety, stress, and depression were $23.79 \pm 9.56,25.35 \pm 10.23$, and $21.88 \pm$ 
TABLE 1: Demographic and clinical characteristics of the participants $(N=293)$.

\begin{tabular}{|c|c|c|c|}
\hline Variables & Mean (SD) & \multicolumn{2}{|c|}{ Patient Activation Measure-13 } \\
\hline Age (yr.) & $63.18(13.44)$ & -0.04 & 0.44 \\
\hline \multirow[t]{2}{*}{ Body mass index } & $22.41(3.28)$ & 0.01 & 0.88 \\
\hline & $N(\%)$ & Statistical test & $P$ value \\
\hline \multicolumn{4}{|l|}{ Gender } \\
\hline Male & $151(51.50)$ & \multirow{2}{*}{$t=-1.01$} & \multirow{2}{*}{0.31} \\
\hline Female & $142(48.50)$ & & \\
\hline \multicolumn{4}{|l|}{ Marital status } \\
\hline Married & $252(86.0)$ & \multirow{2}{*}{$t=-1.80$} & \multirow{2}{*}{0.06} \\
\hline Unmarried+divorce & $41(14.0)$ & & \\
\hline \multicolumn{4}{|l|}{ Educational level } \\
\hline Illiterate & $147(50.2)$ & \multirow{4}{*}{$F=0.24$} & \multirow{4}{*}{0.87} \\
\hline$<$ diploma & $83(28.3)$ & & \\
\hline Diploma & $43(14.7)$ & & \\
\hline Academic & $20(6.8)$ & & \\
\hline \multicolumn{4}{|l|}{ Employment status } \\
\hline Employed & $133(45.4)$ & \multirow{3}{*}{$F=0.02$} & \multirow{3}{*}{0.98} \\
\hline Unemployed & $106(36.2)$ & & \\
\hline Retired & $54(18.4)$ & & \\
\hline \multicolumn{4}{|l|}{ Income (million riyal) } \\
\hline$<0.5$ & $113(38.6)$ & \multirow{4}{*}{$H=2.35$} & \multirow{4}{*}{0.50} \\
\hline $0.5-1$ & $70(23.9)$ & & \\
\hline $1-2$ & $79(27.0)$ & & \\
\hline$>2$ & $31(10.6)$ & & \\
\hline \multicolumn{4}{|c|}{ History of hospital stay (no.) } \\
\hline 0 & $109(37.2)$ & \multirow{5}{*}{$F=1.01$} & \multirow{5}{*}{0.40} \\
\hline 1 & $40(13.7)$ & & \\
\hline 2 & $42(14.3)$ & & \\
\hline 3 & $36(12.3)$ & & \\
\hline$>4$ & $66(22.5)$ & & \\
\hline \multicolumn{4}{|l|}{ Other illnesses } \\
\hline Yes & $186(63.5)$ & \multirow{2}{*}{$t=-0.30$} & \multirow{2}{*}{0.76} \\
\hline No & $107(36.5)$ & & \\
\hline \multicolumn{4}{|l|}{ Drug use } \\
\hline Yes & $98(33.4)$ & & \\
\hline No & $195(66.6)$ & $Z=-1.58$ & 0.11 \\
\hline Diagnosis & & & \\
\hline IHD & $62(21.2)$ & & \\
\hline Diabetes & $55(18.8)$ & & \\
\hline Hypertension & $54(18.4)$ & $F=155$ & 018 \\
\hline $\mathrm{CHF}$ & 45 (15.4) & $F=1.55$ & 0.18 \\
\hline COPD & $41(14.0)$ & & \\
\hline Other $^{\dagger}$ & $33(11.3)$ & & \\
\hline
\end{tabular}

Data were presented numerically (\%). $t=$ independent $t$-test; $Z=$ Mann-Whitney $U$ test; $H=$ Kruskal Wallis test; $F=$ analysis of variance test; IHD = ischemic heart disease; $\mathrm{CHF}=$ congestive heart failure; $\mathrm{COPD}=$ chronic obstructive pulmonary disease. ${ }^{\dagger}$ Chronic kidney disease, multiple sclerosis, rheumatoid arthritis, and cancer.

10.27, respectively. The results showed that $20.9 \%(n=61)$ and $62.0 \%(n=181)$ of the participants had severe and extremely severe anxiety, respectively. $28.3 \% \quad(n=83)$,
$23.5 \%(n=69)$, and $21.8 \%(n=64)$ of the participants had moderate, severe, and extremely severe stress, respectively. In addition, $21.2 \%(n=62), 27.3 \% \quad(n=80)$, and 
TABLE 2: The patient activation measure scores among chronic patients with different levels of anxiety, stress, and depression $(n=293)$.

\begin{tabular}{lcccc}
\hline Variables & Level & $N(\%)$ & $\begin{array}{c}\text { Patient Activation } \\
\text { Measure-13 } \\
\text { Statistical test }\end{array}$ P value \\
\hline \multirow{4}{*}{ Anxiety } & Normal & $6(2.1)$ & & \\
& Mild & $13(4.5)$ & & \\
& Moderate & $31(10.6)$ & $F=0.83$ & 0.73 \\
& Severe & $61(20.9)$ & & \\
Extremely severe & $181(62.0)$ & & \\
& Normal & $44(15.0)$ & & \\
& Mild & $33(11.3)$ & & \\
& Moderate & $83(28.3)$ & $F=1.10$ & \\
& Severe & $69(23.5)$ & & \\
& Extremely severe & $64(21.8)$ & & \\
& Normal & $41(14.0)$ & & \\
& Mild & $35(11.9)$ & & \\
Depression & Moderate & $62(21.2)$ & $F=1.08$ & \\
& Severe & $80(27.3)$ & & \\
& Extremely severe & $75(25.6)$ & & \\
\hline
\end{tabular}

Data were presented numerically (\%).

$25.6 \%(n=75)$ of the participants had moderate, severe, and extremely severe depression, respectively. No significant correlation was observed between anxiety, stress, and depression levels and PAM-13 score $(P>0.05)$ (Table 2).

The mean scores of physical, psychological, and social relationship and environmental subscales of QOL were $44.37 \pm 17.50,48.75 \pm 13.33,46.10 \pm 20.17$, and $48.96 \pm$ 12.65 , respectively. The mean score of general QOL was $47.05 \pm 13.11$.

The bivariate analysis showed that the mean score of PAM-13 was not significantly different according to the demographic and clinical characteristics of the participants (Table 1). A significant positive correlation was observed between all subscales of QOL except the social relationship subscale and PAM-13 $(P<0.05)$. In addition, a significant negative correlation was found between PAM-13, stress, and depression $(P>0.05)$ (Table 3$)$. For further analysis, all variables with $P$ value of $<0.05$ were included in the multiple linear regression analysis. The results of multivariate linear regression with the enter method indicated that only physical and psychological subscales of QOL predicted PAM-13 significantly (Table 4).

\section{Discussion}

The present study investigated the relationship between patient activation, stress, anxiety, depression, and QOL in chronically ill patients admitted to hospital. Based on the results of the study, the PAM score of patients was higher than average, which is not consistent with the results of inpatients and outpatients with chronic diseases in several studies. These studies showed that on average, $15 \%$ of the patients with chronic diseases had the lowest level of activation [21-24]. Methodological differences have played a role in this inconsistency. In this study, patients with chronic diseases were studied by a correlational method, while in other studies, for example, patients with certain types of chronic diseases such as acute coronary syndrome [21] or heart failure [24] were studied longitudinally. In addition, the postdischarge duration can play a role in patient activation. Some studies show that admitted patients or those discharged for less than a month have a higher level of activation [21,25]. Owing to the fact that admitted patients have been studied in this study, a high activation score can be expected.

Many studies have shown that the QOL of chronic patients is negatively associated with depression and anxiety, which was also reflected in the present study, which showed a negative relationship between QOL, depression, and anxiety in cancer patients [26]. Stress also affected the QOL of cancer patients [27]. These results have also been seen in patients on hemodialysis [28] and with coronary heart disease [29]. Therefore, nurses and physicians, the main caregivers of patients, should pay attention to stress, anxiety, and depression in patients that can be effective in their QOL.

According to the results of the present study, a low PAM13 score was associated with a high level of stress and depression; the association was statistically significant. In line with the current study, Magnezi et al., Blakemore et al., and Ahn et al. studied patients referred to primary care clinics [30], the older adults with chronic diseases [31], and patients with osteoarthritis [32], respectively, and found a negative correlation between depression and PAM.

In addition, the Pearson correlation test in the present study showed that a low PAM score was associated with a significant decrease in general QOL and its subscales except social relationship. However, only physical and psychological subscales of QOL significantly predicted the PAM-13. Thus, people better in some subscales of QOL may have higher levels of activation and, consequently, more capacity to participate in self-care behaviors [33, 34]. Erskine et al., who studied patients with acute coronary syndrome, obtained similar results [21]. Since the present study is cross-sectional, the causal relationship between the variables cannot be interpreted. However, depressed patients with lower QOL appear to have lower levels of activation and less involvement in selfmanagement behaviors. Magnezi et al. showed that PAM scores correlated positively with a total Short Form-12 Health Survey (SF-12) score. Magnezi et al. pointed to a cycle in which depressed patients experienced a sense of helplessness and loss of QOL, which in turn is associated with less activation [32]. Therefore, healthcare providers, especially nurses, are expected to design and implement appropriate interventions, for example, psychological and pharmacological interventions [29] and patient support program [35] to reduce the symptoms of depression and improve the QOL in patients with chronic diseases, so they can play an effective role in increasing patient 
TABLE 3: Correlation among the anxiety, stress, depression, quality of life, and patient activation measure in chronic patients $(n=293)$.

\begin{tabular}{|c|c|c|c|c|c|c|c|c|}
\hline Variable & 1 & 2 & 3 & 4 & 5 & 6 & 7 & 8 \\
\hline (1) Anxiety & 1 & & & & & & & \\
\hline (2) Stress & $0.72^{* *}$ & 1 & & & & & & \\
\hline (3) Depression & $0.67^{* *}$ & $0.64^{* *}$ & 1 & & & & & \\
\hline (4) Physical health subscale of QOL & $-0.36^{* *}$ & $-0.32^{* *}$ & $-0.39^{* *}$ & 1 & & & & \\
\hline (5) Psychological subscale of QOL & $-0.16^{* *}$ & -0.11 & $-0.22^{* *}$ & $0.53^{* *}$ & 1 & & & \\
\hline (6) Social relationship subscale of QOL & $-0.26^{* *}$ & $-0.2^{* *}$ & $-0.32^{* *}$ & $0.54^{* *}$ & $0.58^{* *}$ & 1 & & \\
\hline (7) Environmental subscale of QOL & $-0.25^{* *}$ & $-0.18^{* *}$ & $-0.3^{* *}$ & $0.49^{* *}$ & $0.66^{* *}$ & $0.61^{* *}$ & 1 & \\
\hline (8) General QOL & $-0.3^{* *}$ & $-0.26^{* *}$ & $-0.38^{* *}$ & $0.8^{* *}$ & $0.82^{* *}$ & $0.86^{* *}$ & $0.89^{* *}$ & 1 \\
\hline (9) PAM & -0.11 & $-0.13^{*}$ & $-0.13^{*}$ & $0.22^{* *}$ & $0.22^{* *}$ & 0.11 & $0.20^{* *}$ & $0.22^{* *}$ \\
\hline
\end{tabular}

Data were presented as Pearson's correlation coefficient. ${ }^{*} P<0.05 ;{ }^{* *} P<0.01$. QOL $=$ quality of life.

TABLE 4: Predictors of patient activation measure scores by multiple linear regression analysis.

\begin{tabular}{|c|c|c|c|c|c|c|}
\hline \multirow{2}{*}{ Predictors of Patient Activation Measure-13 scores } & \multicolumn{3}{|c|}{ Unstandardized coefficients } & \multirow{2}{*}{$\begin{array}{c}\text { Standardized coefficients } \\
\text { Beta }\end{array}$} & \multirow{2}{*}{$t$} & \multirow{2}{*}{$P$ value } \\
\hline & $B$ & Std. error & $95 \%$ CI for $B$ & & & \\
\hline Constant & 44.92 & 5.11 & & & 8.78 & $<0.001$ \\
\hline Stress & -0.09 & 0.11 & $-0.32-0.13$ & -0.06 & -0.84 & 0.40 \\
\hline Depression & -0.03 & 0.12 & $-0.25-0.20$ & -0.02 & -0.22 & 0.82 \\
\hline Physical health subscale of QOL & 0.21 & 0.10 & $0.02-0.41$ & 0.24 & 2.14 & 0.03 \\
\hline Psychological subscale of QOL & 0.28 & 0.12 & $0.04-0.52$ & 0.24 & 2.28 & 0.02 \\
\hline Environmental subscale of QOL & 0.22 & 0.13 & $-0.04-0.48$ & 0.18 & 1.65 & 0.10 \\
\hline General QOL & -0.39 & 0.24 & $-0.87-0.08$ & -0.34 & -1.64 & 0.10 \\
\hline
\end{tabular}

$\mathrm{CI}=$ confidence interval; $\mathrm{QOL}=$ quality of life.

activation. These interventions ultimately reduce the admission rate of patients [36].

This study had several limitations: longitudinal studies are recommended to identify the causes of PAM change in patients with chronic diseases. Sampling was performed among admitted patients. Therefore, the generalization of results to outpatients should be done with caution. The selfreport results of the patients may not always reflect a valid level of psychological impact, anxiety, stress, and depression of the chronic patients. Therefore, the social desirability bias may affect the results. The specific condition of chronic patients and the long course of treatment can affect the variables examined in these patients. In addition, a large number of questions can also affect patients' answers, which should be used with caution in interpreting the results.

\section{Conclusion}

According to the results of the present study, PAM is less associated with increased stress and depression and decreased some subscales of QOL in patients with chronic diseases. Only the physical and psychological subscales of QOL significantly predicted the level of PAM, so healthcare providers are recommended to plan and implement appropriate interventions to improve the QOL and the healthrelated outcomes.

\section{Data Availability}

The data used to support the findings of this study are included within the article.

\section{Consent}

Consent is not applicable.

\section{Conflicts of Interest}

The authors declare that they have no competing interests.

\section{Acknowledgments}

The authors would like to thank the Non-Communicable Diseases Research Center for its support and collaboration with the Ali Ebne Abitaleb hospital, Rafsanjan University of Medical Science, Rafsanjan, Iran.

\section{References}

[1] R. E. Harris, Epidemiology of Chronic Disease: Global Perspectives, Jones \& Bartlett Learning, 2019.

[2] I. Bos-Touwen, M. Schuurmans, E. M. Monninkhof et al., "Patient and disease characteristics associated with activation for self-management in patients with diabetes, chronic obstructive pulmonary disease, chronic heart failure and 
chronic renal disease: a cross-sectional survey study," PloS One, vol. 10, no. 5, p. e0126400, 2015.

[3] WHO, Chronic Diseases: A Vital Investments, WHO, Geneva, 2008.

[4] G. A. Roth, D. Abate, K. H. Abate et al., "Global, regional, and national age-sex-specific mortality for 282 causes of death in 195 countries and territories, 1980-2017: a systematic analysis for the Global Burden of Disease Study 2017," The Lancet, vol. 392, no. 10159, pp. 1736-1788, 2018.

[5] IHME, What Causes the Most Deaths?, University of Washington, USA, 2019.

[6] J. H. Hibbard, E. R. Mahoney, J. Stockard, and M. Tusler, "Development and testing of a short form of the patient activation measure," Health Services Research, vol. 40, no. 6p1, pp. 1918-1930, 2005.

[7] I. E. O. Moljord, M. L. Lara-Cabrera, L. Perestelo-Perez, A. Rivero-Santana, L. Eriksen, and O. M. Linaker, "Psychometric properties of the patient activation measure-13 among out- patients waiting for mental health treatment: a validation study in Norway," Patient Education and Counseling, vol. 98, no. 11, pp. 1410-1417, 2015.

[8] C. Queenan, K. Cameron, A. Snell, J. Smalley, and N. Joglekar, "Patient heal thyself: reducing hospital readmissions with technology-enabled continuity of care and patient activation," Production and Operations Management, vol. 28, no. 11, pp. 2841-2853, 2019.

[9] J. Greene, J. H. Hibbard, R. Sacks, V. Overton, and C. D. Parrotta, "When patient activation levels change, health outcomes and costs change, too," Health Affairs, vol. 34, no. 3, pp. 431437, 2015.

[10] H. O. Abu, M. D. Anatchkova, N. A. Erskine et al., "Are we "missing the big picture" in transitions of care? Perspectives of healthcare providers managing patients with unplanned hospitalization," Applied Nursing Research, vol. 44, pp. 6066, 2018

[11] J. Greene and J. H. Hibbard, "Why does patient activation matter? An examination of the relationships between patient activation and health-related outcomes," Journal of General Internal Medicine, vol. 27, no. 5, pp. 520-526, 2012.

[12] J. H. Hibbard, J. Greene, and V. Overton, "Patients with lower activation associated with higher costs; delivery systems should know their patients' 'scores'," Health Affairs, vol. 32, no. 2, pp. 216-222, 2013.

[13] E. Titova, Ø. Salvesen, S. B. Bentsen, S. Sunde, S. Steinshamn, and A. H. Henriksen, "Does an integrated care intervention for COPD patients have long-term effects on quality of life and patient activation? A prospective, open, controlled single-center intervention study," PloS One, vol. 12, no. 1, p. e0167887, 2017.

[14] M.-C. R. Goodworth, L. Stepleman, J. Hibbard et al., "Variables associated with patient activation in persons with multiple sclerosis," Journal of Health Psychology, vol. 21, no. 1, pp. 82-92, 2016.

[15] A. B. Harris, F. Kebaish, L. H. Riley, K. M. Kebaish, and R. L. Skolasky, "The engaged patient: patient activation can predict satisfaction with surgical treatment of lumbar and cervical spine disorders," Journal of Neurosurgery: Spine, vol. 32, no. 6, pp. 914-920, 2020.

[16] N. Marsousi, R. A. Farhudi, and A. Lajevardi, "The cultural and social qualiy of life in Iran's cities," Journal of Urban Ecology Researches, vol. 6, no. 12, pp. 79-94, 2016.
[17] C. A. Green, N. A. Perrin, M. R. Polen, M. C. Leo, J. H. Hibbard, and M. Tusler, "Development of the patient activation measure for mental health," Administration and Policy in Mental Health and Mental Health Services Research, vol. 37, no. 4, pp. 327-333, 2010.

[18] S. Nejat, A. Montazeri, K. H. Naieni, K. Mohammad, and S. Majdzadeh, "The World Health Organization quality of Life (WHOQOL-BREF) questionnaire: translation and validation study of the Iranian version," Journal of School of Public Health and Institute of Public Health Research, vol. 4, no. 4, pp. 1-12, 2006.

[19] P. F. Lovibond and S. H. Lovibond, "The structure of negative emotional states: comparison of the depression anxiety stress scales (DASS) with the Beck depression and anxiety inventories," Behaviour Research and Therapy, vol. 33, no. 3, pp. 335-343, 1995.

[20] S. Samani and B. Joukar, "A study on the reliability and validity of the short form of the depression anxiety stress scale (DASS21)," Journal Of Social Sciences And Humanities Of Shiraz University, vol. 26, no. 3, pp. 65-77, 2007.

[21] N. A. Erskine, B. Gandek, M. E. Waring et al., "Survivors of an acute coronary syndrome with lower patient activation are more likely to experience declines in health-related quality of life," The Journal of Cardiovascular Nursing, vol. 33, no. 2, pp. 168-178, 2018.

[22] J. E. Prey, M. Qian, S. Restaino et al., "Reliability and validity of the patient activation measure in hospitalized patients," Patient Education and Counseling, vol. 99, no. 12, pp. 20262033, 2016.

[23] R. L. Skolasky, A. F. Green, D. Scharfstein, C. Boult, L. Reider, and S. T. Wegener, "Psychometric properties of the patient activation measure among multimorbid older adults," Health Services Research, vol. 46, no. 2, pp. 457-478, 2011.

[24] S. M. Dunlay, J. M. Griffin, M. M. Redfield, and V. L. Roger, "Patient activation in acute decompensated heart failure," The Journal of Cardiovascular Nursing, vol. 32, no. 6, pp. 560-567, 2017.

[25] N. F. Eshah, "Acute myocardial infarction survivors experiences: a qualitative literature review," in BITs 2rd Annual World Cancer Congress of Cardiology-2010, pp. 136-136, Taiwan, 2010.

[26] on behalf of the MyBCC Study group, C. G. Ng, S. Mohamed et al., "Anxiety, depression, perceived social support and quality of life in Malaysian breast cancer patients: a 1-year prospective study," Health and Quality of Life Outcomes, vol. 13, no. 1, p. 205, 2015.

[27] M. Dehghan, M. Jazinizade, A. Malakoutikhah et al., "Stress and quality of life of patients with cancer: the mediating role of mindfulness," Journal of Oncology, vol. 2020, Article ID 3289521, 10 pages, 2020.

[28] V. Shafipour, F. Alhani, and A. Kazemnejad, "A survey of the quality of life in patients undergoing hemodialysis and its association with depression, anxiety and stress," Journal of Nursing and Midwifery Sciences, vol. 2, no. 2, pp. 29-35, 2015.

[29] J. M. Moryś, J. Bellwon, S. Höfer, A. Rynkiewicz, and M. Gruchała, "Quality of life in patients with coronary heart disease after myocardial infarction and with ischemic heart failure," Archives of Medical Science: AMS, vol. 2, no. 2, pp. 326-333, 2016.

[30] A. Blakemore, M. Hann, K. Howells et al., "Patient activation in older people with long-term conditions and multimorbidity: 
correlates and change in a cohort study in the United Kingdom," BMC Health Services Research, vol. 16, no. 1, p. 582, 2016.

[31] Y. H. Ahn, B. J. Kim, O. K. Ham, and S. H. Kim, "Factors associated with patient activation for self-management among community residents with osteoarthritis in Korea," Journal of Korean Academy of Community Health Nursing, vol. 26, no. 3, pp. 303-311, 2015.

[32] R. Magnezi, S. Glasser, H. Shalev, A. Sheiber, and H. Reuveni, "Patient activation, depression and quality of life," Patient Education and Counseling, vol. 94, no. 3, pp. 432-437, 2014.

[33] B. X. Ngooi, T. L. Packer, G. Kephart et al., "Validation of the patient activation measure (PAM-13) among adults with cardiac conditions in Singapore," Quality of Life Research, vol. 26, no. 4, pp. 1071-1080, 2017.

[34] M. S. Schmaderer, L. Zimmerman, M. Hertzog, B. Pozehl, and A. Paulman, "Correlates of patient activation and acute care utilization among multimorbid patients," Western Journal of Nursing Research, vol. 38, no. 10, pp. 1335-1353, 2016.

[35] P. M. Shahrbabaki, J. Farokhzadian, and Z. Hasanabadi, "Effect of Self-care Education on Patient's Knowledge and Performance with Heart Failure," Procedia-Social and Behavioral Sciences, vol. 31, pp. 918-922, 2012.

[36] M. J. Shively, N. J. Gardetto, M. F. Kodiath et al., "Effect of patient activation on self-management in patients with heart failure," Journal of Cardiovascular Nursing, vol. 28, no. 1, pp. 20-34, 2013. 